\title{
OnLine Data Management And Interactive Display Software for time series photometry with the ProEM Camera on the McDonald Observatory 82" Otto Struve Telescope
}

\author{
Keaton J. Bell \\ Department of Astronomy and McDonald Observatory, \\ The University of Texas at Austin, Austin, TX 78712, USA \\ E-mail: keatonbastro.as.utexas.edu
}

\begin{abstract}
The OnLine Data Management And Interactive Display (OLD MAID) Software is an all-in-one data reduction and analysis tool for time series photometry. It runs "online," processing the frames as they are acquired and performing aperture photometry to inform observing decisions on the fly. The software produces fully reduced, publication-quality light curves at the conclusion of every observing run and validates the reliability of absolute timestamps. The program also takes care of file management, including the collection of supplementary data products (e.g. local weather data), standardizing digital observing logs, data backup and nightly alerts. Along with the LightField data acquisition software, OLD MAID completes the set of programs required for obtaining high-quality photometric light curves with the ProEM camera at McDonald Observatory's 82" Otto Struve Telescope and beyond. OLD MAID is written in Python and utilizes the crossplatform Qt application framework via PyQt and PyQtGraph. The code and additional details are available at https://github.com/ccd-utexas/OLD-MAID/.
\end{abstract}




\section{Program Summary}

During the acquisition of time series photometry, the OnLine Data Management And Interactive Display (OLD MAID) Software serves the following primary functions (see Figure 1):

- Displays the most recently acquired dark-subtracted, flat-divided frame, allowing the user to select and track target and comparison stars.

- Performs aperture photometry and displays the raw and divided light curves.

- Calculates the Fourier Transform to determine if desired signal-to-noise has been reached.

- Provides an interface for the observer to record comments on the target and conditions.

- Records telescope position, weather conditions, and other metadata.

- Archives reduced frames, photometry, and supplementary data in convenient formats.
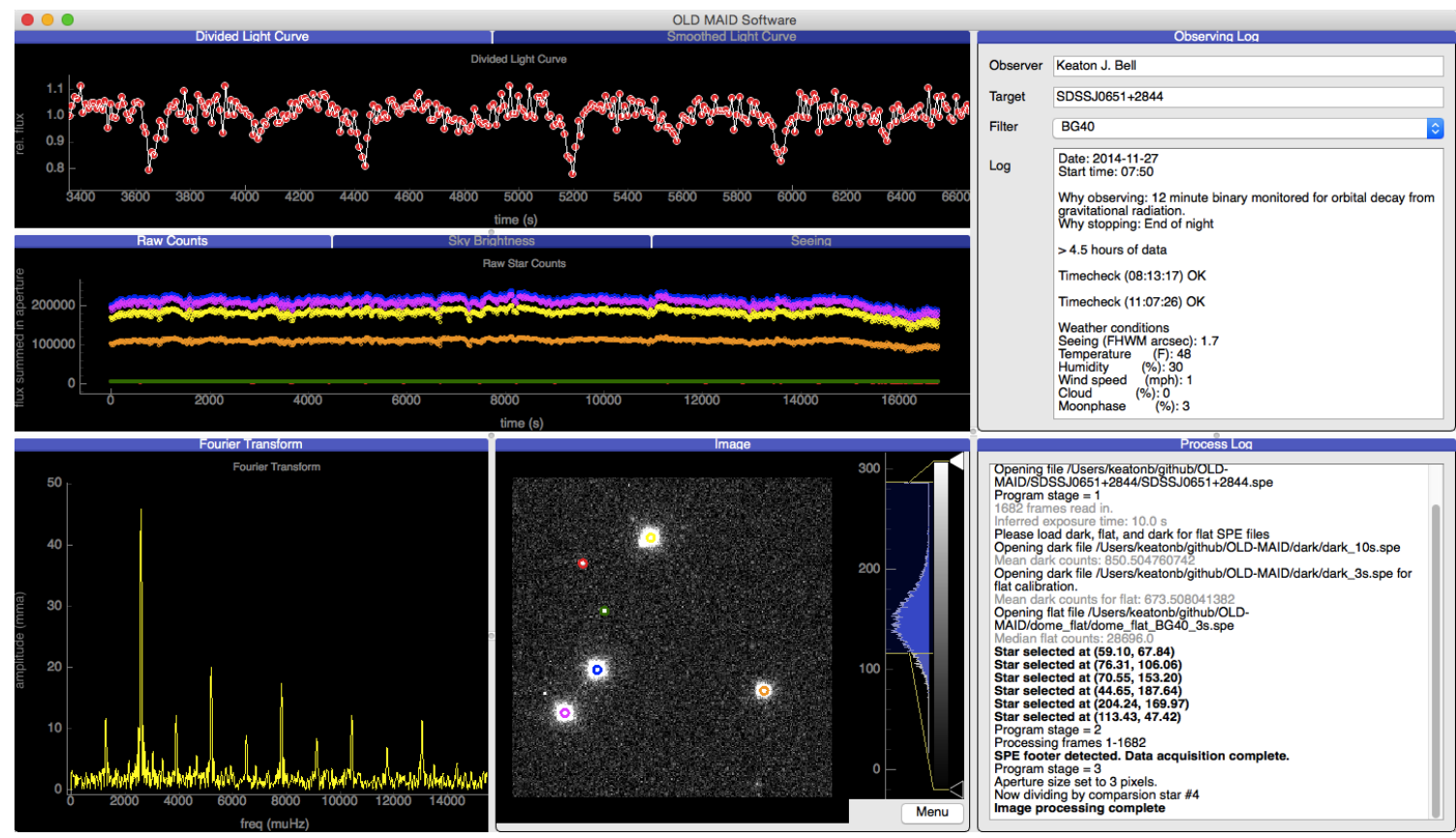

Figure 1: Screenshot of the OLD MAID software in action. The displayed data are from a representative run on the double white dwarf binary system SDSS J0651+2844 (Hermes et al. 2012).

\section{Acknowledgments}

OLD MAID relies on a number of pre-existing software packages. The details of these dependencies are described at https://github.com/ccd-utexas/OLD-MAID/.

\section{References}

[1] Hermes, J. J., Kilic, M., Brown, W. R., et al. 2012, ApJL, 757, L21 